\title{
Zur Theorie der symmetrischen Functionen.
}

\author{
(Von Herrn F. Mertens zu Krakau.)
}

$\mathbf{E}_{\text {sei }}$

$$
f t=\left(t-x_{1}\right)\left(t-x_{2}\right) \ldots\left(t-x_{n}\right)
$$

und $F\left(t_{1}, t_{2}, \ldots t_{n}\right)$ irgend eine ganze rationale Function der $n$ Grössen $t_{1}, t_{2}, \ldots t_{n}$.

Durch successive algebraische Divisionen denke man sich $F\left(t_{1}, t_{2}, \ldots t_{n}\right)$ in die Form gebracht:

$$
F=F_{1} f t_{1}+F_{2} f t_{2}+\cdots+F_{n} f t_{n}+R\left(t_{1}, t_{2}, \ldots t_{n}\right),
$$

wo $F_{1}, F_{2}, \ldots F_{n}, R$ lauter ganze rationale Functionen von $t_{1}, t_{2}, \ldots t_{n}$ sind, von denen überdies die letzte in Bezug auf keine der Variabeln $t_{1}, t_{2}, \ldots t_{n}$ den Grad $n-1$ übersteigt. wie folgt:

Dies vorausgesetzt, zerlege man den Bruch $\frac{R}{f t_{1} f t_{2} \ldots f t_{n}}$ in Partialbrüche,

$$
\frac{R}{f t_{1} f t_{2} \ldots f t_{n}}=\sum \frac{R\left(x_{\alpha}, x_{\beta}, \ldots x_{\nu}\right)}{f^{\prime} x_{\alpha} f^{\prime} x_{\beta} \ldots f^{\prime} x_{\nu}} \frac{1}{t_{1}-x_{\alpha}} \frac{1}{t_{2}-x_{\beta}} \cdots \frac{1}{t_{n}-x_{\nu}},
$$

wo die Indices $\alpha, \beta, \ldots \nu$ unabhängig von einander alle Werthe $1,2,3, \ldots n$ durchlaufen, entwickle beide Seiten dieser Gleichung nach fallenden Potenzen von $t_{1}, t_{2}, \ldots t_{n}$ und vergleiche die Coefficienten von $\left(t_{1} t_{2} \ldots t_{n}\right)^{-1}$. Dies giebt

oder

$$
\left[\frac{R}{f t_{1} f t_{2} \ldots f t_{n}}\right]_{\left(t_{1} t_{2} \ldots t_{n}\right)^{-1}}=\sum \frac{R\left(x_{\alpha}, x_{\beta}, \ldots x_{\nu}\right)}{f^{\prime} x_{\alpha} f^{\prime} x_{\beta} \ldots f^{\prime} x_{\nu}}
$$

$$
\left[\frac{F}{f t_{1} f t_{2} \ldots f t_{n}}\right]_{\left(1_{1} t_{2} \ldots t_{n}\right)^{-1}}=\sum \frac{F\left(x_{\kappa}, x_{\beta}, \ldots x_{\nu}\right)}{f^{\prime} x_{\alpha} f^{\prime} x_{\beta} \ldots f^{\prime} x_{\nu}},
$$

da $R\left(x_{\alpha}, x_{\beta}, \ldots x_{\nu}\right)=F\left(x_{\alpha}, x_{\beta}, \ldots x_{\nu}\right)$ und

ist.

$$
\left[\frac{R}{f t_{1} f t_{2} \ldots f t_{n}}\right]_{\left(t_{1} t_{3} \ldots t_{n}\right)^{-1}}=\left[\frac{F}{f t_{1} f t_{2} \ldots f t_{n}}\right]_{\left(t_{1} t_{3} \ldots t_{n}\right)^{-1}}
$$

Um nun aus der Summe alle Terme zu eliminiren, in denen nicht alle Indices $\alpha, \beta, \ldots \nu$ untereinander verschieden sind, nehme man

$$
F=\Delta . V \text {, }
$$


wo

$$
\begin{aligned}
& \Delta=\quad * \quad\left(t_{1}-t_{2}\right)\left(t_{1}-t_{3}\right) \ldots \ldots\left(t_{1}-t_{n}\right) \times \\
& \left(t_{2}-t_{1}\right) \quad * \quad\left(t_{2}-t_{3}\right) \ldots \ldots\left(t_{2}-t_{n}\right) \times \\
& \left(t_{n}-t_{1}\right)\left(t_{n}-t_{2}\right) \ldots \ldots\left(t_{n}-t_{n-1}\right) *
\end{aligned}
$$

und $V$ eine ganze rationale Function von $t_{1}, t_{2}, \ldots t_{n}$ ist.

Es ist dann

$$
\begin{aligned}
\Delta\left(x_{\alpha}, x_{\beta}, \ldots x_{\nu}\right) & =f^{\prime} x_{\alpha} f^{\prime} x_{\beta} \ldots f^{\prime} x_{\nu} \\
\text { oder } & =0,
\end{aligned}
$$

jenachdem die Indices $\alpha, \beta, \gamma, \ldots \nu$ alle untereinander verschieden sind oder nicht, und somit

$$
\Sigma V\left(x_{\alpha}, x_{\beta}, \ldots x_{\nu}\right)=\left[\frac{\Delta V}{f t_{1} f t_{2} \ldots f t_{n}}\right]_{\left(t_{1} t_{2} \ldots t_{n}\right)^{-1}},
$$

wo jetzt die Summe alle Werthe umfasst, welche $V$ durch Permutation der $x_{1}, x_{2}, \ldots x_{n}$ annehmen kann.

Ist nun $V$ eine symmetrische Function, so sind alle diese Werthe einander gleich, und man hat

$$
V\left(x_{1}, x_{2}, \ldots x_{n}\right)=\frac{1}{n !}\left[\frac{\Delta V}{f t_{1} f t_{2} \ldots f t_{n}}\right]_{\left(t_{1} t_{2} \ldots t_{n}\right)^{-1}} .
$$

Da der Entwickelungscoefficient auf der rechten Seite nur von den in $f t$ und $V$ vorkommenden Coefficienten abhängt, so stellt derselbe somit einen independenten Ausdruck der Function $V$ durch die Coefficienten der Gleichung $f t=0$ vor.

Krakau, im October 1868. 